\title{
SPIN LIGHT IN NEUTRINO TRANSITION BETWEEN DIFFERENT MASS STATES
}

\author{
A. GRIGORIEV * \\ Skobeltsyn Institute of Nuclear Physics, Moscow State University, \\ 119991 Moscow, Russia \\ A. $\mathrm{LOKHOV}^{\dagger}$ \\ Department of Quantum Statistics and Quantum Field Theory, Moscow State \\ University, \\ 119991 Moscow, Russia \\ A. STUDENIKIN \\ Department of Theoretical Physics, Moscow State University, \\ 119991 Moscow, Russia \\ A. TERNOV § \\ Department of Theoretical Physics, Moscow Institute for Physics and Technology, \\ 141700 Dolgoprudny, Russia
}

\begin{abstract}
The spin light of neutrino is considered in the process of a neutrino radiative transition between two different mass states in presence of medium. By this study we investigate the influence of background matter on the initial and final neutrino states in the process of massive Dirac neutrino decay due to the non-zero transition magnetic moment. We derive corresponding corrections to the total width of the process over the matter density in most important for applications cases.
\end{abstract}

\section{Introduction}

Electromagnetic properties of neutrino are among the key items of modern particle physics (see ${ }^{1}$ for a recent review). It seems quite natural that a massive neutrino would have nonzero diagonal or transition magnetic

*ax.grigoriev@mail.ru

†lokhov.alex@gmail.com

${ }^{\ddagger}$ studenik@srd.sinp.msu.ru

§a_ternov@mail.ru 
moment. If a neutrino has non-trivial electromagnetic properties, then neutrino coupling to photons is possible and several important, for instance for astrophysics, processes can exist ${ }^{2}$. Recently we have proposed a new mechanism of neutrino radiation of photons that is realized when a relativistic neutrino with nonzero magnetic moment moves in dense matter. This mechanism was termed the "spin light of neutrino" $(S L \nu)^{3}$. The quantum theory of this phenomenon was developed in ${ }^{4}$ (see also ${ }^{5}$ and ${ }^{6}$ ).

In this paper we extend our studies of $S L \nu^{4}$ and consider the $S L \nu$ in a more general case when the photon is emitted in the neutrino radiative decay. The $S L \nu$ considered in ${ }^{4}$ was investigated under condition of equal masses for the initial and final neutrino states. Here below we examine the case when the neutrino transition between two different neutrino mass states is realized. Thus, we consider the $S L \nu$ mode in the neutrino radiative decay in matter originated due to the neutrino transition magnetic moment.

It should be noted that the neutrino radiative decay was considered before by several authors ${ }^{7}$. It was shown that the process characteristics are substantially changed if the presence of a medium is taken into account. In these calculations, the influence of the background matter was considered only in the electromagnetic vertex. Here we are going to discuss the impact of the medium also onto the state of neutrino itself. At the same time we will be interested in the another aspect of the problem and consider it from the point of view of light emission. Under the condition of equal initial and final particle masses the process becomes equivalent to the $S L \nu$ in matter. With different masses for the initial and final neutrino states, the spin light becomes only the constituting channel for the overall process corresponding to the change of the neutrino helicity. The mechanism of $S L \nu$ is based on helicity states energy difference of the particle arising due to the weak interaction with the background matter. Hence, our study makes sense, obviously, if the scale of neutrino mass difference is of the order of spin energy splitting owing to the interaction with matter.

Let us specify now the process under consideration. We are considering the decay of one neutrino mass state $\nu_{1}$ into another mass state $\nu_{2}$ assuming that $m_{1}>m_{2}$, and restrict ourselves with only these two neutrino species and accordingly with two flavour neutrinos. Having in mind that conditions for the most appropriate application of the process under study can be found in the vicinity of neutron stars we will take for the background a neutron-rich matter. In this case a process with participation of antineutrinos is more appropriate and thus will study here. However for the convenience in what follows we will still refer to the particles as 
to neutrinos. Since the interactions of flavour neutrinos with neutron star matter are the same and governed by the neutron density we will take equal interactions for the initial and final massive neutrinos with the matter.

\section{Modified Dirac equation}

The system "neutrino $\Leftrightarrow$ dense matter" depicted above can be circumscribed mathematically in different ways. Here we use the powerful method of exact solutions, discussed in a series of our papers ${ }^{6}$. This method is based on solutions ${ }^{4}$ of the modified Dirac equation for neutrino in the background medium

$$
\left\{i \gamma_{\mu} \partial^{\mu}-\frac{1}{2} \gamma_{\mu}\left(1+\gamma^{5}\right) f^{\mu}-m\right\} \Psi(x)=0,
$$

where in the case of unpolarized and nonmoving matter $f^{\mu}=G_{f} / \sqrt{2}(n, \mathbf{0})$ with $n$ being matter number density. At this, the energy spectrum of neutrino is given by

$$
E_{\varepsilon}=\varepsilon \sqrt{\left(p-s \alpha m_{\nu}\right)^{2}+m_{\nu}^{2}}+\alpha m_{\nu}, \quad \alpha=\frac{1}{2 \sqrt{2}} G_{F} \frac{n}{m_{\nu}}
$$

where $\varepsilon= \pm 1$ defines the positive and negative-energy branches of the solutions, $s$ is the helicity of neutrino, $p$ is the neutrino momentum. The exact form of the solutions $\Psi_{\varepsilon, p, s}(\mathbf{r}, t)$ can be found in ${ }^{4}$ and ${ }^{6}$.

\section{Spin light mode of massive neutrino decay}

The S-matrix element for the decay has the standard form that of the magnetic moment radiation process:

$$
S_{f i}=-(2 \pi)^{4} \mu \sqrt{\frac{\pi}{2 w L^{3}}} \delta\left(E_{2}-E_{1}+w\right) \delta^{3}\left(\mathbf{p}_{2}-\mathbf{p}_{1}+\mathbf{k}\right) \bar{u}_{f}\left(\mathbf{e}, \boldsymbol{\Gamma}_{f i}\right) u_{i} .
$$

Here $\boldsymbol{\Gamma}=i \omega\left\{[\boldsymbol{\Sigma} \times \varkappa]+i \gamma^{5} \boldsymbol{\Sigma}\right\}, u_{i, f}$ are the spinors for the initial and final neutrino states, $\mathbf{e}$ is the photon polarization vector, $\mu$ is the transitional magnetic moment ${ }^{1}$ and $L$ is the normalization length.

In the process, we have the following conservation laws:

$$
E_{1}=E_{2}+\omega ; \quad \mathbf{p}_{\mathbf{1}}=\mathbf{p}_{\mathbf{2}}+\mathbf{k} .
$$

It is useful to carry out our computations through non-dimensional terms. For that purpose we introduce the following notations: $\gamma=\frac{m_{1}}{p_{1}} ; \kappa=\frac{\alpha m_{1}}{p_{1}}=$ $\frac{\tilde{n}}{p_{1}} ; \delta=\frac{\Delta m^{2}}{p_{1}^{2}}=\frac{m_{1}^{2}-m_{2}^{2}}{p_{1}^{2}}$. To single out the the spin light part of the radiative decay process we should choose different helicities for the initial and final neutrinos. Keeping the analogy with the usual process of $S L \nu$ we take the 
helicity quantum numbers as $s_{1}=-1, s_{2}=1$. Then the solution of the kinematic system (4) can be written in the form

$$
w=\frac{-\left(K D+x \kappa^{2}\right)+\sqrt{\left(K D+x \kappa^{2}\right)^{2}-\left(K^{2}-\kappa^{2}\right)\left(D^{2}-\kappa^{2}\right)}}{\left(K^{2}-\kappa^{2}\right)}
$$

with the notations $D=s_{1} \kappa-\delta ; \tilde{n}=\frac{1}{2 \sqrt{2}} G_{F} n, K=\sqrt{\left(1-s_{1} \cdot \kappa\right)^{2}+\gamma^{2}}-x$, here $x$ stands for $\cos \theta, \theta$ is the angle between $\mathbf{p}_{1}$ and $\mathbf{k}$.

Performing all the calculations we obtain angle distribution of the probability for the investigated process:

$$
\frac{d \Gamma}{d x}=\mu^{2} p_{1}^{3} \frac{(K-w+x)(w K-\kappa-\delta) w^{3} S^{\prime}}{\sqrt{(K D-w+x)^{2}-\left(K^{2}-\kappa^{2}\right)\left(D^{2}-\kappa^{2}\right)}},
$$

where $S^{\prime}=\left(1+\beta_{1} \beta_{2}\right)\left(1-\frac{w-x-w \cdot x+w \cdot x^{2}}{\sqrt{1+w^{2}-2 w \cdot x}} x\right)-\left(\beta_{1}+\beta_{2}\right)\left(x-\frac{w-x}{\sqrt{1+w^{2}-2 w \cdot x}}\right)$ and $\beta_{1}=\frac{1+\kappa}{\sqrt{(1+\kappa)^{2}+\gamma^{2}}}, \beta_{2}=\frac{\sqrt{1+w^{2}-2 w \cdot x}-\kappa}{K-w+x}$.

The total probability can be computed from the equation (6) by taking the integration over the angle $\theta$ range. However, manual calculations are not quite simple to carry through them. Even though the integral can be calculated exactly, the final expression is enormously complex and its explicit form is optional to be given here.

\section{Results and discussion}

It is worth to investigate the asymptotical behavior of the probability $\Gamma$ in three most significant relativistic limiting cases keeping only the first infinitesimal order of small parameters. On this way we have,

$$
\begin{gathered}
\Gamma=4 \mu^{2} \tilde{n}^{3}\left(1+\frac{3}{2} \frac{m_{1}^{2}-m_{2}^{2}}{\tilde{n} p_{1}}+\frac{p_{1}}{\tilde{n}}\right),\left(\text { ultrahigh density: } 1 \ll \frac{p_{1}}{m_{1}} \ll \frac{\tilde{n}}{p_{1}}\right) ;(7) \\
\Gamma=4 \mu^{2} \tilde{n}^{2} p_{1}\left(1+\frac{\tilde{n}}{p_{1}}+\frac{m_{1}^{2}-m_{2}^{2}}{\tilde{n} p_{1}}+\frac{3}{2} \frac{m_{1}^{2}-m_{2}^{2}}{p_{1}^{2}}\right),\left(\text { high density: } \frac{m_{1}^{2}}{p_{1}^{2}} \ll \frac{\tilde{n}}{p_{1}} \ll 1\right) ;
\end{gathered}
$$

$$
\Gamma \approx \mu^{2} \frac{m_{1}^{6}}{p_{1}^{3}}, \text { (quasi-vacuum case: } \frac{\tilde{n}}{p_{1}} \ll \frac{m_{1}}{p_{1}} \ll 1, m_{1} \gg m_{2} \text { ). }
$$

The obtained results (7) and (8) exhibit the power of the method of exact solutions since they establish clear connection between the case of massive weak-interacting particles when the masses of the initial and final particles differ with the previously investigated equal mass case. Indeed, it is easy to verify that these results transforms exactly into the results of $S L \nu$ calculation. ${ }^{3}$ The asymptotic estimation (9) can not be reduced to the 
$S L \nu$ case and thus it is a new result, which is characteristic feature for the decay process under study. The above-mentioned asymptotical cases (7), (8) and (9) where calculated with the assumption that the initial neutrino is relativistic $\left(\gamma=\frac{m_{1}}{p_{1}} \ll 1\right)$. In particular the relativistic character of the initial neutrino propagation influences strongly on the emitted $S L \nu$ photon energy because of increase of the part of neutrino energy in it.

It is also interesting to investigate the spin light mode in the radiative decay of slowly moving massive neutrino (or even stationary initial neutrino). This process has been calculated several times ${ }^{7}$. We consider the vacuum case to find the interrelation of our results obtained using the method of exact solutions with the results of previous works. So, taking into account $\gamma=\frac{m_{1}}{p} \ll 1, \kappa=\frac{\tilde{n}}{p_{1}}, \delta \equiv \frac{\gamma^{2}}{2}$ for the probability of the process we finally get:

$$
\Gamma \approx \frac{7}{24} \mu^{2} m_{1}^{3} \sim m_{1}^{5} .
$$

We obtain here the same dependency of the probability from the mass of the decaying neutrino as in the classical papers on the radiative neutrino decay. By this means we justify usage of the modified Dirac equation exact solutions method.

Acknowledgements. One of the authors (A.S.) is thankful to Kim Milton for the invitation to attend the 9th Conference on Quantum Field Theory Under the Influence of External Conditions (Oklahoma, USA) and for the kind hospitality provided in Norman.

\section{References}

1. C. Giunti, A. Studenikin, Phys.Atom.Nucl.72, 2151, 2009.

2. G. Raffelt, Stars as Laboratories for Fundamental Physics, (Univ. of Chicago Press, 1996); G. Raffelt, Phys. Rep. 320, 319 (1999).

3. A. Lobanov and A. Studenikin, Phys.Lett.B564, 27 (2003), A. Lobanov and A. Studenikin, Phys.Lett.B601, 171 (2004).

4. A. Studenikin, A. Ternov, Phys.Lett.B608, 107 (2005), A. Grigorev, A. Studenikin, A. Ternov, Phys.Lett.B622, 199 (2005).

5. A. Lobanov Phys.Lett.B619, 136 (2005).

6. A. Studenikin, J. Phys. A: Math. Gen.39, 6769 (2006); A. Studenikin, J. Phys. A: Math. Theor. 41, 164047 (2008).

7. S. Petcov, Sov.J.Nucl.Phys 25, 340 (1977), G. T. Zatsepin, A. Yu. Smirnov, Yad. Fiz. 28, 1569 (1978) Yad. Fiz.28, 1569 (1978), P.B. Pal and L. Wolfenstein, Phys.Rev.D25, 766 (1982), J.C.D'Olivo, J.F.Nieves, and P.B. Pal, Phys.Rev.Lett.64, 1088 (1990), C. Giutni, C.W. Kim and W.P. Lam, Phys.Rev.D43, 164 (1990), A. Ternov, P. Eminov J.Phys.G:Nucl.Part.Phys.29, 357 (2005). 